\title{
Heterogeneity of population of microorganisms grown in presence of iron oxide maghemite nanoparticles
}

\author{
Tatyana P. Denisova ${ }^{1, *}$, Elena V. Simonova ${ }^{2}$, Lubov A. Kokorina ${ }^{2}$, Evgenia N. Maximova ${ }^{1}$, Oleg M. Samatov ${ }^{3}$, \\ Alexander P. Safronov ${ }^{3,4}$, Galina V. Kurlyandskaya ${ }^{4,5}$ \\ ${ }^{1}$ Irkutsk State University, Nizhnyaya Embankment St., 6, Irkutsk, 664011, Russia \\ ${ }^{2}$ FSBEI HE Irkutsk State Medical University MOH, Red Revolt str., 1, Irkutsk, 664003, Russia \\ ${ }^{3}$ Institute of Electrophysics, Amundsen str., 106, Ekaterinburg, 620016, Russia \\ ${ }^{4}$ Ural Federal University, Mira St. 19 Ekaterinburg, 620000, Russia \\ ${ }^{5}$ Universidad del País Vasco UPV-EHU, Leioa, 48940 Spain
}

\begin{abstract}
In this work $\gamma-\mathrm{Fe}_{2} \mathrm{O}_{3}$ MNPs were obtained by laser target evaporation and water based suspensions were prepared. Maximum permissive dose of iron in water (MPD) is $0.3 \mathrm{mg} / \mathrm{L}$. It was found that 100 MPD dose of iron induces formation of non-typical colonies after 72 or 96 hours exposition: against a background of small black colonies large white colonies appeared due to a disruption in tyrosine synthesis. Multiple re-cloning of the white colonies grown with MNPs showed that they retained their properties both under standard conditions (temperature of $24{ }^{\circ} \mathrm{C}$ ) and at the temperatures up to $37^{\circ} \mathrm{C}$. E.nigrum grown with MNPs demonstrated very scant extension of small colonies at the cultivation temperature of $24^{\circ} \mathrm{C}$, their growth was completely blocked at $37^{\circ} \mathrm{C}$. Significant changes in the structure of the population were noted. First of all, large cells with pronounced aggregation were observed among the black colonies. These aggregates consisted of large cells connected to each other by matrix. In the white colonies the appearance of very long threadlike cells connecting different groups of the cells establishing an intercellular communication was evident. $\mathrm{Fe}_{2} \mathrm{O}_{3}$ MNPs induce an increase in the heterogeneity of the population, expressed as a change in morpho-physiological states.
\end{abstract}

\section{Introduction}

The universal property of any microbial population showing its adaptive capability is the heterogeneity. The study of this phenomenon is of interest for understanding the development of the microbial population in conditions of stress arising from the unfavourable environmental factors, as well as the mechanism of "quorum sensing", which provides a way of the microorganisms behaviour and survival in the microbial community [1]. It is known that heterogeneity in microbial populations can be discrete and continuous, arising due to phenotypic or genotypic variability [2]. Heterogeneity in the structure of microbial population is determined by factors of interaction between the external and internal factors, and also due to the variability of their physiological and biochemical properties. Heterogeneity provides microbial communities chance to survive in changing living conditions.

Many living systems, including microbial populations strongly depend on iron in variety of life processes [3]. Therefore, consideration that variation of small amount of iron is an instrument of the study of heterogeneity in the biological samples can provide useful insight on the features of the development of microbial population in artificially created stress conditions.
Exophiala nigrum (E. nigrum, black unicelular yeast) of the Baykal Lake play an important role in the equilibrium of this unique ecosystem [4]. Black yeasts were previously used as a model system for the estimation of cytotoxicity, MNPs accumulation and inductive heat capacity studies of the biological samples grown in the presence of MNPs [5]. For fabrication of spherical iron oxide magnetic nanoparticles (MNPs) electrophysical technique of the laser target evaporation (LTE) was employed [6].

The aim of the work was to study the heterogeneity in the structure of the microbial population of Exophilia nigrum under the influence of controlled amount of iron provided by iron oxide $\gamma-\mathrm{Fe}_{2} \mathrm{O}_{3}$ nanoparticles in form of electrostatically stabilized water-based suspension.

\section{Experimental}

Iron oxide MNPs were synthesized by the laser target evaporation technique. In the LTE installation, the solid target (compacted commercial $\mathrm{Fe}_{2} \mathrm{O}_{3}$ powder from Alfa Aesar) is overheated by the high-energy pulse of a focused laser beam. Rapid evaporation in the laser spot area is a result of beam energy transfer into the kinetic energy of the vapours, ejected away from the surface of 
the target made of the. More details can be found elsewhere [6-7].

Structural studies of LTE MNPs were performed by transmission electron microscopy (TEM, JEOL JEM2100 microscope operating at $200 \mathrm{kV}$ ). The X-ray diffraction (XRD) studies were performed by DISCOVER D8 (Bruker) diffractometer using $\mathrm{Cu}-\mathrm{K} \alpha$ radiation with wave lengths $\lambda=1.5418 \AA$. The stoichiometric ratio of $\mathrm{Fe}^{2+} / \mathrm{Fe}^{3+}$ in all samples of the iron oxide MNPs was determined based on the analysis of XRD diffractograms, and redox potentiometric titration using an automatic TitroLine titrator.

Electrostatically stabilized suspensions based on $\gamma$ $\mathrm{Fe}_{2} \mathrm{O}_{3}$ LTE MNPs were prepared by ultrasound treatment using sodium citrate solutions $(5 \mathrm{mM})$ in distilled water. A Hermle Z383 centrifuge operated at $10.000 \mathrm{rpm}$ for $10 \mathrm{~min}$ was used in order to remove large aggregates. Magnetic measurements were carried out on a MPMS XL-7 SQUID-magnetometer.

As a test culture, the strain R-11 Exophiala nigrum, belonging to the saprophyte forms of black yeast, was isolated from the natural population by streaking on a solid nutrient medium for yeast cultivation. Strain has a black colour due to the produced melanin. For experimental studies, a test culture was used in the exponential stage of growth. The R-11 strain was registered in the All-Russian Collection of Microorganisms of the Research Institute of Genetics No. Y 309. It can be used as a destructor of the main components of wastewater from the cellulose and paper industry (lignin, in particular) [8-9]. For both scanning (SEM) and transmission (TEM) electron microscopy cell cultures were prepared by standard procedure [10].

Maximum permissive dose of iron ions in water (MPD) is $0.3 \mathrm{mg} / \mathrm{L}$. Electrostatically stabilized suspension of MNPs was used for preparation of solutions for biological part of the experiments on the basis of Saburo liquid nutrient medium in concentration 100 MPD of iron. First, a cell suspension of yeast was prepared, which was used for all studied cases including control. Next, the flasks were prepared with MNPs suspensions for studied concentrations (liquid medium Saburo + MNPs). In the control case Saburo medium had no MNPs. After this, the same volume of the same cell suspension of the test culture was added to the prepared liquid medium Saburo + MNPs, after which the mixture was vigorously shaken, and then the bite was sewn onto a solid nutrient medium. The control samples were grown with Saburo liquid medium and corresponding addition of a sterile physiological solution. The exposure was 72 and 96 hours at a temperature of $24^{\circ} \mathrm{C}$. In the control version, instead of suspension of MNPs an equivalent saline volume was added. Exposed for 72 , and 96 hours samples were used in order to determine the properties of the E. nigrum cultures. For this purpose, the yeast was sown to a solid nutrient medium. The smear preparations were also elaborated, staining them with gentianviolet. The heterogeneity of the microbial population was evaluated under the experimental conditions for the following indicators: growth of culture on a solid nutrient medium and change in the morphological features of cells that are specific for each microorganism species under standard conditions.

\section{Results and discussion}

Fig. 1 shows the as-prepared spherical LTE MNPs. XRD studies gave a mean crystallite size for MNPs of $19 \pm 2$ $\mathrm{nm}$ in a good agreement with TEM data. The chemical composition found by redox titration was close to the stoichiometric maghemite $\left(\mathrm{Fe}_{2.72} \mathrm{O}_{4}\right)$.
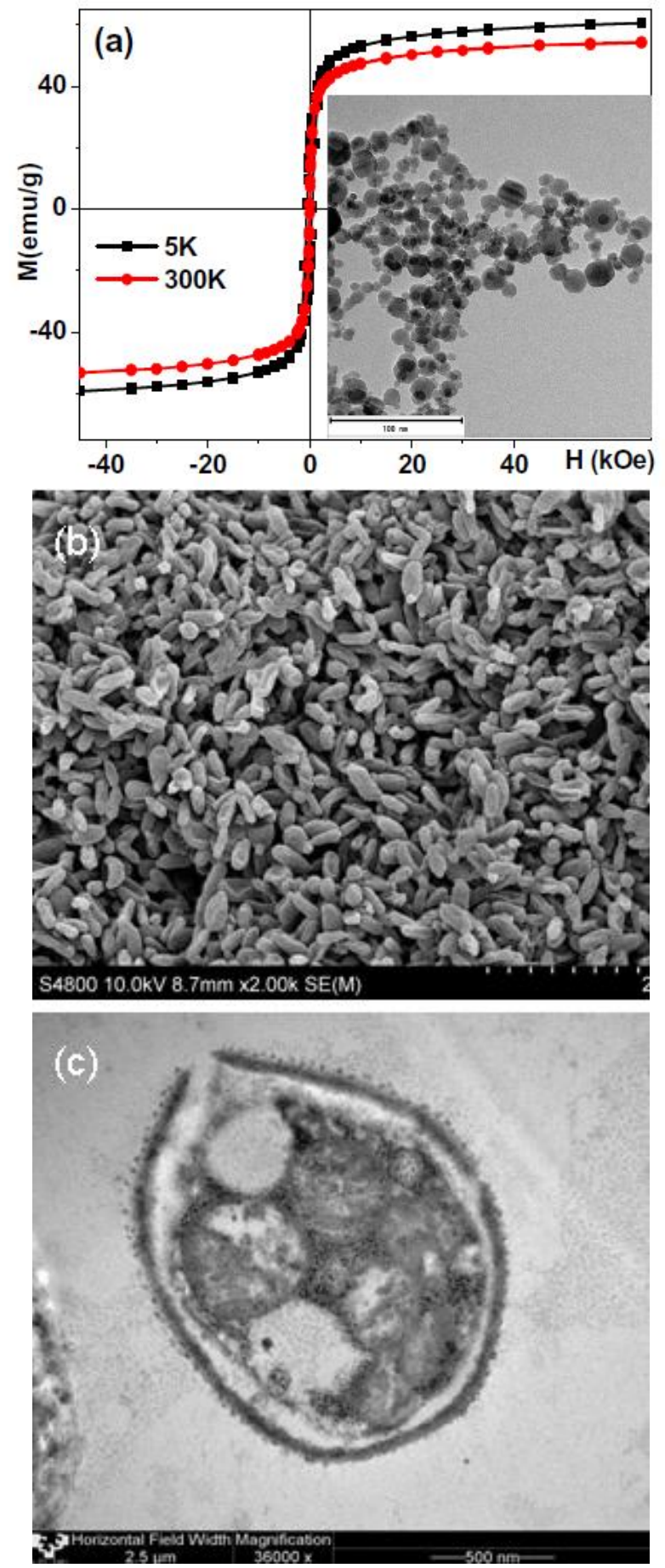

Fig. 1. Hysteresis loops of as-prepared spherical LTE MNPs of iron oxide (a) Inset shows TEM image of MNPs. E. Nigrum cell cultures grown without MNPs: SEM (b) and TEM (c). 
One can see that hysteresis loops of MNPs show no saturation at both temperatures: the magnetization in the field of $65 \mathrm{kOe}$ in both cases is close to $54 \mathrm{emu} / \mathrm{g}$ for 300 $\mathrm{K}$ and $61 \mathrm{emu} / \mathrm{g}$ for $5 \mathrm{~K}$ temperature. The comparative analysis of structural and magnetic parameters confirms consistency of obtained data about close to superparamegnetic behaviour for MNPs of this size.

Fig. 1(b) shows general view of E. Nigrum cell cultures grown in the presence of 0 MPD of iron: individual cell size and morphologies were typical for $E$. nigrum. It was found that culturing the E. nigrum test culture on Saburo solid nutrient medium without MNPs results in good grows of the culture with the formation of colonies typical for this strain. When MNPs were added in iron concentration of 100 MPD for 72 and 96 hours cultivation, a change in the properties of the cell cultures was observed. Namely, the large white colonies appeared on the background of very small E. nigrum colonies of black colour (Fig. 2).

White colonies grown without MNPs onto the Saburo nutrient medium did not alter their properties after several times re-cloning both under standard growth conditions (the cultivation temperature of $24^{\circ} \mathrm{C}$ ) and with an increase in the cultivation temperature up to 37 ${ }^{\circ} \mathrm{C}$. At the same time, very low growing rate of the black colonies, grown up without MNPs was observed at the cultivation temperature of $24^{\circ} \mathrm{C}$ and their growth was completely blocked at $37^{\circ} \mathrm{C}$ temperature. White colony color is associated with a breakdown in the synthesis of melanin, and the root cause may be a violation of any stage of the biochemical cycle of tyrosine, from which the precursors of melanin are formed. The MNPs amount corresponding to $100 \mathrm{MPD}$ of iron change biochemical properties of black yeasts leading to a disruption of the synthesis of melanin in the E. nigrum strain R-11 culture.

It is known that E. nigrum strain R-11, grown without MNPs is a compactly organized uniform cell mass. The degree of heterogeneity of the morphotype is poorly expressed. During the time the cells were in liquid medium Saburo + MNPs (1 min), no changes were found (taking into account the number of cells in $1 \mathrm{ml}$, the number of colonies grown latter onto a solid medium and the morphology of the colonies).

At the same time, significant changes in the structure of the microbial population were noted in culture grown with MNPs for the exposure of 72 or 96 hours. First of all, among the black colonies of the $E$. nigrum strain R-11, morphotypes (Fig. 2) of large cells were isolated with their pronounced aggregation and connected to each other by the matrix. Such a morphotype is typical feature of a static culture, for which the concentration of cells ceases to increase due to a decrease in the growth rate, depending on a number of factors, such as low partial pressure of oxygen or the content of toxic substances in the nutrient medium. In the culture of the microbial population of E. nigrum, which had grown in the form of white colonies, along with ordinary cells, very long threadlike cells appeared connecting different groups of cells in course of intercellular communication (Fig. 3). The presence of local aggregates of cells, interconnected by the intercellular matrix, was detected. The formation of such a morphotype is also associated with a violation of the development of microbial culture: the biomass of the cell increases and the processes of its division do not occur. This indicates that biofilm formation around the MNP is likely to occur as a result of the process of adaptation to unfavourable living conditions. Comparing the character of the change in the properties of cell culture and morphological properties of E. nigrum strain R-11 grown without MNPs, it should be noted that the observed degree of heterogeneity under experimental conditions is the result of its reserve of stability, as a result of which physiologically active cells die in unfavourable environmental conditions, whereas, physiologically inactive forms are able to survive.
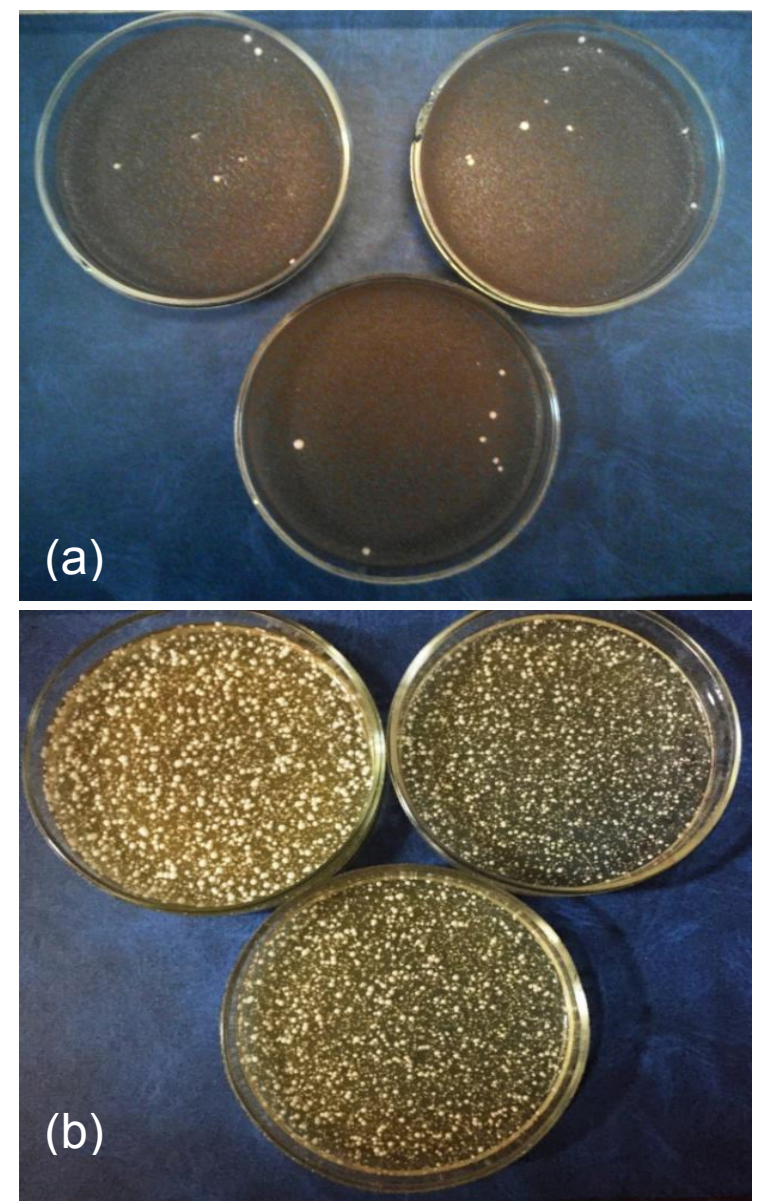

Fig. 2. Colonies of yeast of white colour in the experiment with $10^{\prime}$ ) i 4 PD addition of iron in the form of iron oxide MNPs: at exposures of 72 hours (a) and 96 hours (b).

Although careful magnetic characterization of iron oxide MNPs was discussed above at this study we did not provide a magnetic characterization of cell cultures grown in the presence of MNPs. At the same time obtained results on the heterogeneity of the microbial population and activation of the mechanism of a biofilm formation as a reaction on the presence of LTE MNPs in nutrient give new insight on our previous results [11], where total reflection x-ray fluorescence spectroscopy and magnetic measurements were used as power tools for evaluation of iron concentration in ferrofluids and yeast samples. Observed surprisingly low amount of iron 

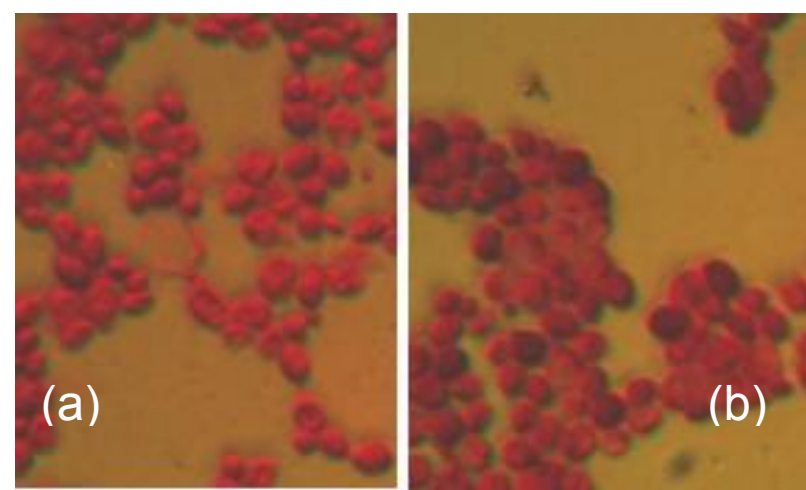

Fig. 3. Morphology of melanin-containing cells grown on a solid medium in the presence of LTE MNPc in concentration corresponding to 100 MPD of iron: (a) - 72 hours exposire, (b) - 96 hours exposure.
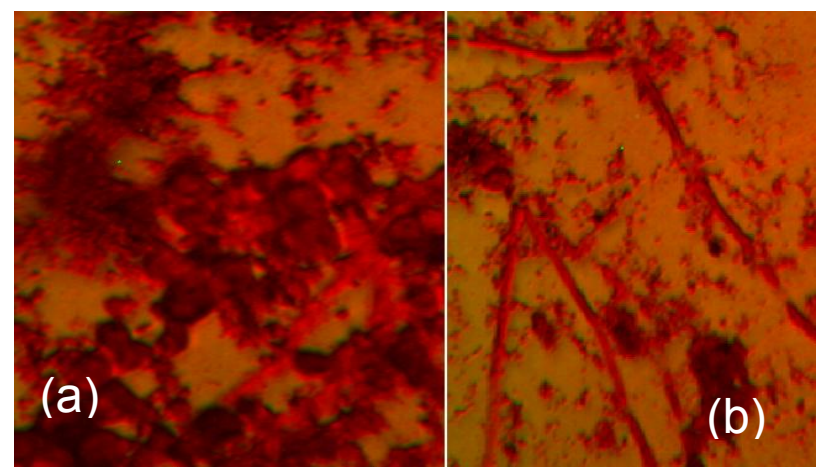

Fig. 4. Morphology of E. nigrum, cells grown on a solid medium. in the presence of LTE MNPc in concentration corresponding to $100 \mathrm{MPD}$ of iron forming white colonies: (a) - 72 hours exposire, (b) - 96 hours exposure.

in the yeast samples grown in the presence of LTE MNPs was previously given a suggestion that membrane absorption can be proposed as a possible mechanism of MNPs incorporation. Here we observed that biofilm formation around the MNP is likely to occur. This additional barrier can also contribute to the low degree of iron MNPs incorporation.

The next step in the studies of the process of adaptation of $E$. nigrum to unfavourable living conditions and better understanding of the mechanisms of iron accumulation would be application of external magnetic field (both static and alternating) during cell cultivation and comparative studies of the iron content and magnetic responses. MNPs containing model samples with different concentration of magnetic nanoparticles are highly requested by a new interdisciplinary field - development of magnetic biosensors [12]. To be reliable the main features of the cell cultures with different amount of MNPs must be similar and therefore heterogeneity of population of microorganisms is important part of the development of this research line.

\section{Conclusions}

Exophiala nigrum strain R-11 unicelular organisms of the Baikal Lake were used for heterogeneity of population analysis. Yeasts were grown in the presence of $19 \pm 2 \mathrm{~nm}$ maghemite MNPs produced by laser target evaporation. Electrostatically stabilized water based suspensions were prepared for biological experiments to study the ability of iron oxide nanoparticles at a concentration equivalent to $100 \mathrm{MPD}$ of iron to induce a change in the structure of a microbial population.

It has been established that under the influence of the iron oxide nanoparticles at a concentration equivalent to $100 \mathrm{MPD}$ of iron the heterogeneity of the population of saprophytic eukaryotic microorganisms was increased in comparison with the control case (grown without MNPs). A change in the morpho-physiological and biochemical characteristics of cells of E. nigrum strain R-11 induced by iron oxide nanoparticles was detected. Heterogeneity ensures the stability of the microbial population and allows it to remain viable using the mechanism of a biofilm formation.

The work was supported by the Ministry of Education and Science of the Russian Federation (project No. 3.6121.2017) and RFFI №16-34-50192 grants. We thank R. Andrade, Iu. Novoselova and I.V. Beketov for special support. Selected measurements were made at SGIKER services UPV/EHU.

\section{References}

1. M.B. Miller, B.L. Bassler Quorum sensing in bacteria. Annu Rev Microbiol. 55, 199 (2001).

2. L.A. Magdanova, N.V. Golyasny. Microbiology. 82, 3 (2013).

3. M. Ilbert and V. Bonnefoy, Biochimica et Biophysica Acta - Bioenergetics, 1827, 161 (2013).

4. I.P. Babieva and I.Yu. Chernov, Biology of yeasts (M.V. Lomonosov MSU, Moscow, 2004).

5. J.P. Novoselova, A.P. Safronov, O. Samatov, I.V. Beketov, H. Khurshid, Z. Nemati, H. Srikanth, T. P. Denisova, R. Andrade, and G.V. Kurlyandskaya, IEEE Trans. Magn. 50, 4600504 (2014).

6. A.P. Safronov, I.V. Beketov, S.V. Komogortsev, G.V. Kurlyandskaya, A.I. Medvedev, D.V. Leiman, A. Larrañaga, and S.M. Bhagat, AIP Adv., 3, 052135 (2013).

7. V.V. Osipov, V.V. Platonov, M.A. Uimin, and A.V. Podkin, Tech. Phys. 57, 543 (2012).

8. V. V. Pavlenko, V. M. Kharlamov, V. I. Chemerilova, S. S. Timofeeva. SU 1071637 A, C 12 N 15/00// C 02 F 3/34; C 12 R 1/00.

9. S.S.Pavar, V.V. Pavlenko. Messenger of IrGSHA. 60, 67 (2014).

10. G.V. Kurlyandskaya, Iu.P. Novoselova, V.V. Schupletsova, R. Andrade, N.A. Dunec, L.S. Litvinova, A.P. Safronov, K.A.Yurova, N.A. Kulesh, A.N. Dzyuman, and I.A. Khlusov 431, 249 (2017).

11. N.A. Kulesh, I.P. Novoselova, A.P. Safronov, I.V. Beketov, O.M. Samatov, G.V. Kurlyandskaya, M.V. Morozova, and T.P. Denisova, J. Magn. Magn. Mater. 415, 39 (2016).

12. G.V. Kurlyandskaya, E. Fernandez, A.P. Safronov, A.V. Svalov, I.Beketov, A. Burgoa Beitia, A. GarciaArribas, F.A. Blyakhman, Appl. Phys. Lett. 106 193702 (2015). 